\title{
COMUNICAÇÃO DA EQUIPE MULTIPROFISSIONAL E INDIVÍDUOS PORTADORES DE HIPERTENSÃO ARTERIAL*
}

\author{
Magda Cristina Queiroz Dell'Acqua** \\ Janete Pessuto*** \\ Sílvia Cristina Mangini Bocchi*** \\ Renata Cristina Peloso Macero dos Anjos****
}

DELL'ACQUA, M.C.Q.; PESSUTO, J.; BOCCHI, S.C.M.; ANJOS, R.C.P.M. Comunicação da equipe multiprofissional e indivíduos portadores de hipertensão arterial. Rev.latino-am.enfermagem, Ribeirão Preto, v. 5, n. 3, p. 43-48, julho 1997.

O presente estudo tem como objetivos levantar dados sobre o que os indivíduos portadores de hipertensão arterial pensam sobre a sua doença e verificar a ocorrência do processo de comunicação entre estes e os profissionais que atuam junto aos mesmos. Foram entrevistados 66 clientes, obtendo-se 73 narrativas, constatando-se que a maioria (69 narrativas) dos participantes da pesquisa faz correlação com o conceito de hipertensão arterial, ainda que de maneira pouco elaborada. Apesar de $95 \%$ dos clientes fazerem alguma referência à doença, não significa que as orientações recebidas anteriormente foram aprendidas. Espera-se que o enfermeiro por desempenhar um importante papel dentro da equipe multiprofissional, identifique as possíveis barreiras relativas ao processo de comunicação, havendo daí a intervenção junto aos elementos que podem estar dificultando ou impedindo a compreensão necessária para que o cliente assuma seu autocuidado.

UNITERMOS: comunicação em saúde, hipertensão arterial, fatores de risco

\section{INTRODUÇÃO}

As doenças cardiovasculares são a primeira causa de óbitos no país. A hipertensão arterial atinge cerca de $10 \%$ da população adulta, diminuindo a expectativa média de vida do portador dessa patologia, devido à insuficiência cardíaca, insuficiência vascular cerebral, coronária e renal $^{3,4,11}$.

Um indivíduo é considerado portador de hipertensão arterial quando a pressão arterial diastólica permanece acima de $90 \mathrm{~mm}$ de $\mathrm{Hg}$ e a sistólica acima de

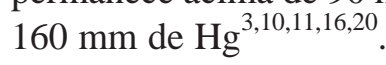

Existem alguns fatores, denominados fatores de risco, que podem influenciar no aparecimento da hipertensão arterial essencial, sendo divididos em dois grupos: e sexo $6,7,12,16,17$

1.1- CONGÊNITOS: hereditariedade, idade, raça

1.2- ADQUIRIDOS: obesidade, alimentação rica em sal e gorduras, álcool, tabaco, drogas anticoncepcionais, estresse $\mathrm{e}^{2,7,8,16,17,20}$.
Para haver uma correta abordagem junto ao indivíduo portador de hipertensão arterial, tem-se que considerar, além dos fatores de risco, os seguintes aspectos: situação sócio-econômica; grau de instrução; atividade que executa; sentimentos e conhecimento sobre a doença; crenças de saúde; estilo de vida; experiência anterior com a doença no meio em que vive; percepção da seriedade do problema; complexidade do tratamento; atividades decorrentes do sistema de saúde vigente; efeitos colaterais dos medicamentos; percepção social do problema; relacionamento inadequado com membros da equipe de saúde ${ }^{14}$.

A comunicação, instrumento básico na relação enfermeiro-paciente, merece destaque neste processo para que seja efetiva, pois, embora vários autores considerem a sua importância na prática profissional, a mesma tem sido vista como um canal pouco explorado e ineficiente ${ }^{18}$.

Existem alguns fatores que são considerados como barreiras no processo de comunicação. São eles: - limitação do emissor ou receptor - quando há problemas relacionados com a audição, visão, não havendo

\footnotetext{
* Apresentado no $5^{\circ}$ SIBRACEn

** Auxiliar de Ensino do Curso de Graduação em Enfermagem da Faculdade de Medicina de Botucatu - UNESP - SP, Brasil *** Professor Assistente do Curso de Graduação em Enfermagem da Faculdade de Medicina de Botucatu - UNESP - SP, Brasil **** Enfermeira responsável pela Seção de Saúde Pública, do Ambulatório do Hospital das Clínicas da Faculdade de Medicina de Botucatu - UNESP - SP, Brasil
} 
compreensão da mensagem ou estímulo emitido. Pode ocorrer devido a problemas de natureza orgânica, memória, atenção ou raciocínio;

- pressuposição da compreensão - quando a pessoa que orienta crê que a mensagem foi entendida e não fornece mais explicações sobre o assunto abordado;

- imposição de esquema de valores - as crenças e os valores do cliente devem ser respeitados;

- ausência de significação comum- o cliente não entende a linguagem do transmissor;

- influência de mecanismos inconscientes- quando o cliente nega sua doença, julgando-se sadio ${ }^{19}$.

Uma das etapas do tratamento da hipertensão arterial é o chamado tratamento não-farmacológico, que consiste de medidas terapêuticas nas quais os indivíduos são orientados sobre a doença, o tratamento e a mudança em seu estilo de vida, para que a pressão arterial possa ser controlada. Nessa fase, a motivação do indivíduo, para que participe do tratamento de forma efetiva, é fundamental $^{5,8,9,12}$.

Somente medidas de orientação não bastam para que os sujeitos mudem seu comportamento. São necessários métodos que contemplem as necessidades de conhecer, para que os clientes incorporem às suas vidas atitudes que contribuam com o controle da doença. As medidas educacionais devem ser contínuas, e os indivíduos devem ser vistos como únicos, com seus problemas, sua história de vida, para que as causas de não-adesão ao tratamento sejam atingidas ${ }^{13}$.

Os métodos de ensino-aprendizagem são: instrução individual- ideal para o levantamento dos dados do cliente, treino de habilidades, sendo mais utilizada na fase inicial, onde há a intensificação do autocuidado, e também onde se constrói a motivação e se faz o "feedback" daquilo que já foi orientado; ensino em grupooportunidade de aprendizagem através da experiência de vários indivíduos, facilitando também a atuação do educador, no sentido de atingir um número maior de clientes para assuntos comuns aos mesmos ${ }^{15}$.

\section{OBJETIVOS}

2.1- Identificar junto a uma clientela portadora de hipertensão arterial o que pensam ser essa doença.

2.2- Verificar a ocorrência do processo de comunicação entre tal clientela e os profissionais que atuam junto a mesma.

\section{METODOLOGIA}

\subsection{Tipo de Estudo}

Trata-se de uma pesquisa descritiva, onde foi priorizado o discurso dos entrevistados, como fonte de informação, para a análise do conceito de hipertensão arterial junto aos portadores.

A origem deste estudo deu-se a partir dos diálogos desenvolvidos durante estratégia educativa com clientes portadores de hipertensão arterial, partindo da premissa de que tudo o que é dito ou escrito é passível de ser submetido à análise de conteúdo. Para análise temática utilizou-se de técnica proposta por BARDIN ${ }^{1}$.

\subsection{Caracterização do campo de pesquisa e da população}

Este trabalho foi realizado junto ao Centro de Hipertensão Arterial (C.H.A.), onde realizam-se atendimentos ambulatoriais a hipertensos em um Hospital Universitário do interior paulista. Neste Centro os pacientes recebem atendimento multiprofissional, terapêutico e educativo de enfermeiros, médicos, nutricionistas, assistentes sociais e alunos da graduação médica e de enfermagem.

O C.H.A. funciona semanalmente, às quintasfeiras, no período da manhã, sendo que os indivíduos são atendidos conforme consultas agendadas.

\section{3 - Sujeitos Particulares}

Participaram deste estudo sessenta e seis sujeitos, portadores de hipertensão arterial, clientes do ambulatório, que freqüentaram os grupos de orientação.

\section{4 - Procedimentos}

A coleta de dados ocorreu durante as atividades educativas, coordenada pela enfermeira, por um período de dois meses. Desta forma, os portadores de hipertensão arterial tiveram oportunidade para participarem de discussões e debates multilaterais (Figura 1) sobre a fisiopatologia, o tratamento e seus hábitos.

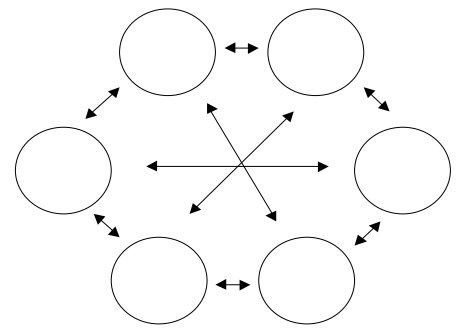

*****Figura 1 - Comunicação multilateral entre enfermeiro e clientes 
Essa estratégia educativa tende a facilitar a participação, comunicação de experiências e dúvidas, além de favorecer a tomada de decisões para o autocuidado dos sujeitos, quanto ao tratamento da doença. Para dinamizar tal atividade o enfermeiro utilizou-se de uma questão norteadora para a condução do grupo, sendo: - "O que é hipertensão arterial para vocês?"

Tal questão foi levantada antes do início das orientações, sendo que as opiniões dos participantes foram registradas na íntegra, constituíndo o corpus (conjunto das transcrições), que foi submetido à análise transversal. Esse procedimento analítico envolveu o recorte do corpus ao redor de cada unidade de registro, destacadas através de leituras das falas dos sujeitos e, posteriormente, ordenadas pela classificação em seis subcategorias.

\subsection{Definição de categoria e subcategorias}

Neste estudo a categoria e subcategorias emergiram da própria leitura das unidades de registro, subsidiadas na técnica de análise de conteúdo ${ }^{1}$.

As unidades de registro foram classificadas e agregadas em subcategorias, permitindo a análise das ocorrências, sendo os seguintes critérios utilizados para interpretação dos significados:

C1 - associação com fatores de risco: obesidade, estresse, alimentação rica em cloreto de sódio e gorduras, diabetes mellitus, hereditariedade;

C2 - associação com complicações: complicações em órgãos-alvo como coração, rins, olhos e cérebro;

C3 - associação parcial: são os conceitos que não manifestaram a plenitude do significado proposto pela literatura, que considera hipertensão arterial para a pressão arterial diastólica, valores permanentes acima de $90 \mathrm{~mm}$ de $\mathrm{Hg}$ e para a sistólica, acima de $160 \mathrm{~mm}$ de $\mathrm{Hg}^{10,11}$;

C4 - associação com sintomas: distúrbios da visão, sudorese e calafrios, cefaléia occiptal, arritimia cardíaca, formigamentos;

C5 - não associa: aquelas que referenciavam não saber; C6 - associação com medidas terapêuticas: exercícios.

$\mathrm{O}$ estabelecimento desses critérios permitiu a construção do corpo de categoria e subcategorias especificamente para este estudo.

Quadro 1 - Corpo de categoria e subcategorias: códigos e definições

\begin{tabular}{|l|l|}
\hline \multicolumn{1}{|c|}{ CATEGORIAE SUBCATEGORIAS } & \multicolumn{1}{c|}{ DEFINIÇÕES } \\
\hline categoria C & não conceitua \\
\hline - sub-categoria C1 & - associação com fatores de risco \\
\hline - sub-categoria C2 & - associação com complicações \\
\hline - sub-categoria C3 & - associação parcial \\
\hline - sub-categoria C4 & - associação com sintomas \\
\hline - sub-categoria C5 & - não associa \\
\hline - sub-categoria C6 & - associação com medidas terapêuticas \\
\hline
\end{tabular}

\section{6 - Tratamento Estatístico}

Os dados foram analisados descritivamente, com indicação de freqüências absolutas e relativas pertencentes a cada subcategoria, possibilitando a interpretação e análise.

\section{RESULTADOS E DISCUSSÃO}

O Quadro 2 apresenta as 73 unidades de registro, referentes aos conceitos de hipertensão arterial manifestados pelos sujeitos, classificadas em categoria única, denominada não conceitua. 
Quadro 2 - Frequiência de respostas às unidades de registro, referentes aos conceitos de hipertensão arterial manifestados pelos sujeitos, segundo categoria e subcategorias. Botucatu, 1995

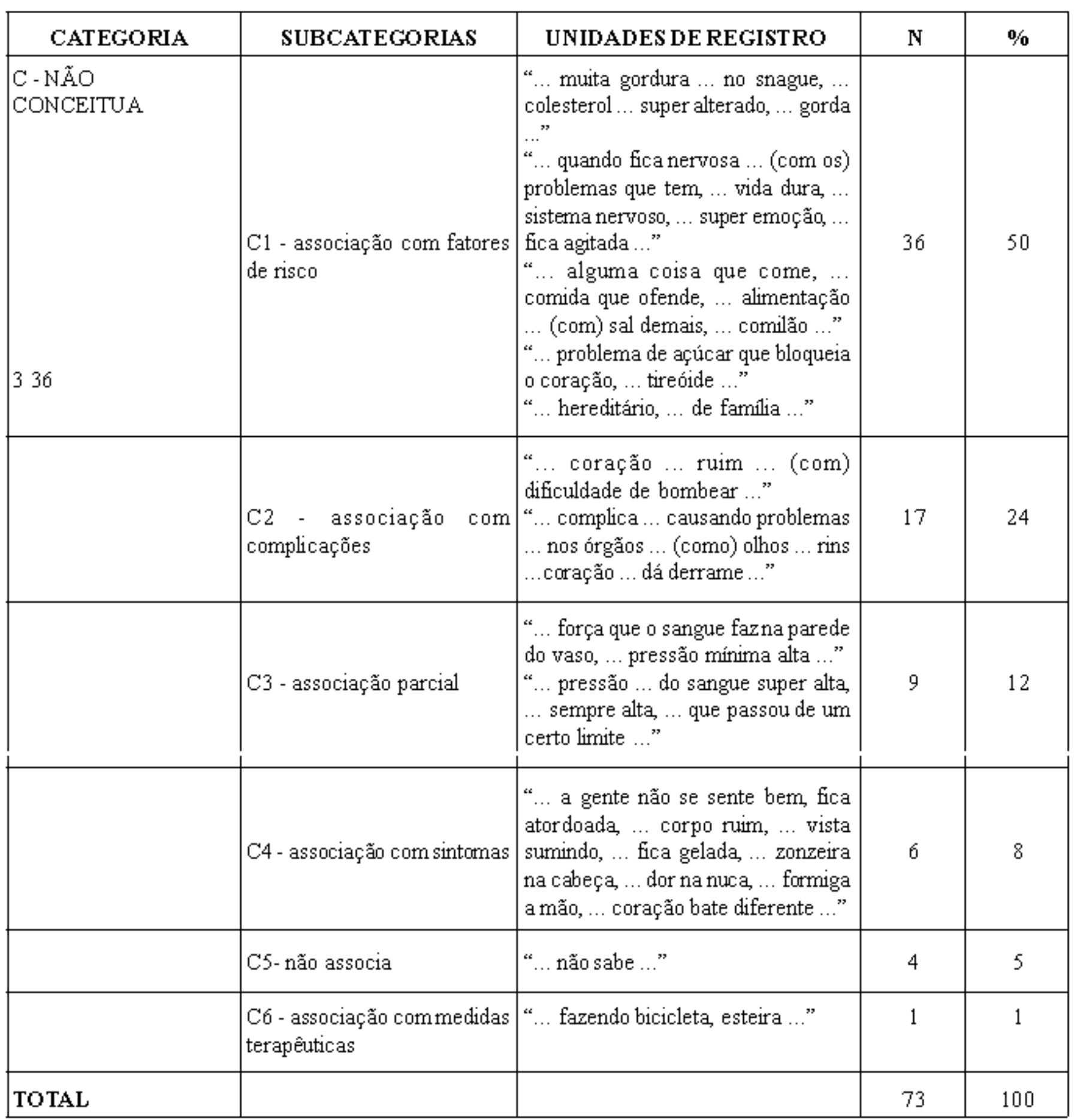

Percebe-se a dificuldade dos portadores de hipertensão arterial em conceituar a doença, porém a grande maioria procura associá-la a algo. Desta forma, emergem 6 subcategorias onde $50 \%$ delas estão associadas com fatores de risco; $24 \%$ com complicações; $12 \%$ fazem associação parcial; $8 \%$ com sintomas; $5 \%$ não associam e $1 \%$ associa com medidas terapêuticas.

$\mathrm{Na}$ subcategoria $\mathrm{C} 1$ as narrativas revelam que existe uma correlação com os fatores de risco congênitos e adquiridos, estando de acordo com a literatura pesquisada,correspondendo a 36 citações $(50 \%)^{3,5,7,11,16,17,20}$.

Na subcategoria C2 existem 17 narrativas, sendo que $24 \%$ associam o conceito de hipertensão arterial às complicações. Tais relatos estão de acordo com alguns autores que citam como complicações dessa patologia o comprometimento de órgãos-alvo, como coração, rins e cérebro $^{3,4,7,11,16}$

$\mathrm{Na}$ subcategoria C3 $12 \%$ das citações demonstram uma associação denominada de parcial, pois, 
embora não se enquadrem no conceito encontrado na literatura pesquisada, são alguns dos aspectos abordados em tal definição ${ }^{3,5,7,10,11,16}$.

$\mathrm{Na}$ subcategoria $\mathrm{C} 4$ têm-se $6 \%$ das narrativas, onde há relação do conceito de hipertensão arterial com sintomas. Esta doença é assintomática, porém uma parcela pequena de portadores dessa patologia pode apresentar algumas queixas ${ }^{9,16}$.

A subcategoria C5 demonstra que 5\% dos sujeitos não sabem o que é hipertensão arterial, podendo tais respostas estarem associadas às barreiras de comunicação, já que quando o indivíduo é cadastrado no Serviço, tem oportunidade de ser avaliado por diversos profissionais, recebendo todas as orientações necessárias sobre a doença ${ }^{19}$.

$\mathrm{Na}$ subcategoria C6 somente uma narrativa referiu-se às medidas terapêuticas, demonstrando que não há clareza em relação ao conceito de hipertensão arterial e o que vem a ser o tratamento.

Ao se verificar a efetividade do processo de comunicação entre a equipe multiprofissional e a clientela deste estudo, percebe-se que, através das narrativas, a maioria dos indivíduos apresentou conceitos vagos que até puderam ser associados à definição de hipertensão arterial, apesar de estarem pouco elaborados.

Salienta-se que, nesse Serviço, a maior parte dos clientes cadastrados já teve alguma oportunidade de receber informações sobre a patologia e sobre o tratamento, seja em consulta médica, consulta de enfermagem, atendimento nutricional ou leitura de boletins informativos que, eventualmente, são distribuídos, abordando aspectos básicos sobre a doença.

A dificuldade de elaborar conceitos mais complexos que denotem a apreensão, por parte dos sujeitos, do significado dos assuntos abordados, também foi relatada em outro estudo, que demonstrou que a maior parte dos indivíduos portadores de hipertensão arterial, nesse mesmo serviço, quando fizeram referências sobre o que sabiam da doença e do tratamento, citaram que basearam suas respostas na experiência própria de cada um, não fazendo menção às orientações que já haviam recebido em várias situações. Quando questionados se as orientações que recebiam eram suficientes, $84,3 \%$ da amostra responderam afirmativamente. Porém, anteriormente, referiram que a base de conhecimento para tais respostas era a experiência própria ${ }^{14}$.

\section{5- CONSIDERAÇÕES FINAIS}

Como estratégia para realizar a educação em saúde, uitilizou-se a comunicação multilateral, onde o enfermeiro, apesar de coordenador do grupo, ao mesmo tempo integrou-se a ele. Com isso, pretendeu-se que o processo de comunicação ocorresse de forma interativa, dando oportunidade aos indivíduos para participarem e emitirem seus conceitos sobre hipertensão arterial.

Todavia, não se pode deixar de citar a questão das barreiras à comunicação, pois, apesar de somente 5\% da amostra não apresentarem nenhuma associação com o conceito de hipertensão arterial, o fato de $95 \%$ fazerem algum tipo de abordagem referente à doença, não significa que as orientações recebidas anteriormente tenham sido efetivas, já que os conceitos apreendidos foram incompletos.

Considerando-se a comunicação como um instrumento básico para a assistência de enfermagem e, percebendo-se que existem dificuldades que podem afetar tal processo, espera-se que o enfermeiro, por desempenhar um papel importante na equipe multiprofissional, tenha a intencionalidade de detectar tais barreiras, podendo assim intervir nos elementos que estejam dificultando ou impedindo a compreensão básica efetiva necessária à tomada de decisão para o autocuidado.

\section{COMMUNICATION BETWEEN THE HEALTH STAFF AND HYPERTENSIVE PATIENTS}

This study aims to analyse data on hypertensive individuals and their concern about the disease as well as to verify the communication process that took place between the individuals and the health staff working with them. Sixty-six individuals were interviewed and we obtained 73 statements. The majority of them (69 narratives) expressed correlation to high blood pressure concepts even though in a poorly elaborated form. Although $95 \%$ of the patients referred to the illness, it does not mean that the orientations were understood. Nurses are expected to perform an important role in the multiprofessional team, identifying possible obstacles to the process of communication in order to enable the clients' comprehension and their self care. 


\section{COMUNICACIÓN ENTRE LOS PROFESIONALES DE LA SALUD Y LOS PORTADORES DE HIPERTENSIÓN ARTERIAL}

Este trabajo tiene como objetivo coger datos sobre lo que piensan los portadores de hipertensión arterial sobre su enfermedad y verificar el proceso de comunicación entre los mismos y con los profesionales de la salud que actuan junto a ellos. Fueron entrevistados 66 clientes, obteniendo 73 narrativas, de las cuales se constató que la mayoría (69 narrativas) de los sujetos hicieron referencia al concepto de hipertensión arterial, aunque de forma pocoelaborada. A pesar de que el $95 \%$ de los clientes hacieron alguna referencia a la enfermedad, no significa que las orientaciones recibidas anteriormente fueron aprehendidas. Se espera que el enfermero, por desempenãr un importante papel dentro del equipo multiprofesional, identifique las barreras posibles del proceso de comunicación, obteniendo al mismo que interviene en las etapas que pueden estar dificultando o impidiendo la comprensión necesaria para que cliente asuma su autocuidado.

TÉRMINOS CLAVES: comunicación en salud, hipertensión arterial; factores de riesgo

\section{REFERÊNCIAS BIBLIOGRÁFICAS}

01. BARDIN, L. Análise de conteúdo. Lisboa, Persona, 1977.

02. BRANDÃO, M.R.F.; MATSUDO, V.K.R. Stress, emoção e exercício. Rev.Bras.Ciênc.Mov., v.4, p. 95-9, 1990.

03. BRASIL. Ministério da Saúde. Secretaria de Assistência à Saúde. Departamento de Programas de Saúde. Coordenação de Doenças Cardiovasculares. Controle da hipertensão arterial: uma proposta de integração ensinoserviço. Rio de Janeiro: MS/SAS/DPS/CDCVMEC/UFRJ/NUTES, 1993

04. BRASIL. Ministério da Saúde. Secretaria Nacional de Programas Especiais de Saúde. Divisão Nacional de Doenças Crônico-Degenerativas. Guia para controle da hipertensão arterial. Brasília: Centro de Documentação do Ministério da Saúde, 1983.

05. CARVALHO, J.J.M. Aspectos preventivos em cardiologia. Arq. Bras. Cardiol., v. 50, p. 5967, 1988.

06. FRANCISCHETTI, E. et al. Hipertensão arterial leve: o que além das cifras? Arq.Bras.Cardiol., v. 52, p. 349-65, 1989.

07. HOLLENBERG, N.K. Hypertension: mechanisms and therapy. In: BRAUNWALD, E. Atlas of heart diseases. Philadelphia: Current Medicine, 1994. v. 1.

08. JOINT NATIONAL COMMITTEE ON DETECTION, EVALUATION AND TREATMENT OF HIGH BLOOD PRESSURE. The fifth report. Arch. Intern. Med., v. 153, p. 154-83, 1993.

09. KOCHAR, M.S.; WOODS, K.D. Controle da hipertensão: para enfermeiras e demais profissionais de saúde. 2. ed. São Paulo: Andrei, 1990.
10. MARANHÃO, M.F. de C.; RAMIRES, J.A.F. Aspectos atuais do tratamento da hipertensão arterial. Arq. Bras. Cardiol., v. 51, p. 99-105, 1988.

11. MILlER, O. Diagnóstico e terapêutica em medicina interna. 14. Ed. Rio de Janeiro, Atheneu, 1988. Cap. 30, p. 643-82: Doenças cardiovasculares.

12. MION JÚNIOR, D. Hipertensão: aspectos práticos São Paulo: Sociedade Brasileira de Cardiologia; Departamento de Hipertensão Arterial, 1988.

13. PESSUTO, J. As necessidades básicas afetadas e os fatores de risco de clientes portadores de hipertensão arterial. Ribeirão Preto, 1994. 158 p. Dissertação (Mestrado) - Escola de Enfermagem de Ribeirão Preto - USP.

14. PIERIN, A.M.G.; CAR, M.R. Instrumento de consulta de enfermagem a pessoas com hipertensão arterial em tratamento ambulatorial. Rev. Esc. Enfermagem USP, v. 26, p. 17-32, 1992.

15. RANKIN, S.H.; STALLINGS, K.D. Patient education: issues, principles, practices. 2. ed. Philadelphia: J. B. Lippincott, 1990. cap. 7, p. 165-90: Planning: client goals in patient education.

16. RIBEIRO, A.B. Atualização em hipertensão arterial: clínica, diagnóstico e terapêutica. São Paulo: Atheneu, 1996.

17. SCHMIEDER, R.E. et al. Riesgos en el desarrollo de hipertensión arterial. Medicina (Buenos Aires), v. 48, p. 303-10, 1988.

18. SILVA, M.J.P. da. O toque e a distância interpessoal entre enfermeiros e pacientes nas consultas de enfermagem. Rev. Esc. Enfermagem USP, v.25, p.309-18, 1991.

19. STEFANELLI, M.C. Comunicação com paciente: teoria e ensino. $2^{\text {a }}$ ed. São Paulo: Robe, 1993.

20. STUART, E.M.; FRIEDMAN, R.; BENSON, H. Promoting nonpharmacologic interventions to treat elevated blood pressure. 